\title{
De la crítica a la sociología conflacionista al realismo crítico morfogenético en Margaret Archer
}

\section{From criticism to conflationist sociology to morphogenetic critical realism in Margaret Archer}

Sergio Pignuoli-Ocampo (spignuoli@conicet.gov.ar) Facultad de Ciencias Sociales, Universidad de Buenos Aires (Buenos Aires, Argentina) ORCID: 0000-0002-9918-0931

\begin{abstract}
This text reconstructs the general criticism to conflation formulated by Margaret Archer. In its social morphogenesis programme, Archer elaborates an acute diagnosis about the main deficits of different constructions of sociological object and she questioned them ontological and epistemologically through the figure of conflation, in which she distinguished three modes (downwards, upwards and central). Here we reconstruct each of them separately and we analyse the formulation in nuce the theoretical basis of critical and morphogenetic realism, a scientific research programme that she establishes as a nonconflation sociological way, supported by critical realism and analytical dualism. In doing so, we assume the systematic perspective of multileveled research programmes and the category of metatheorizing as prelude by Ritzer.
\end{abstract}

Key words: morphogenesis, conflation, realism, dualism, practice, social.

\section{Resumen}

Este trabajo reconstruye la crítica general del conflacionismo hecha por Margaret Archer. En su programa de morfogénesis social la autora británica realizó un agudo diagnóstico acerca de los déficits fundamentales de las diversas construcciones del objeto sociológico y las cuestionó teórica y epistemológicamente a través de la figura crítica de conflacionismo, en la que distinguió tres modalidades (descendente, ascendente y central). Aquí las reconstruiremos por separado y analizaremos en esa crítica general la formulación in nuce de las bases teóricas o fundamento operativo del programa de realismo crítico morfogenético, que impulsará la autora con el ariete alternativo de establecer una sociología noconflacionista, informada ontológica y epistemológicamente por el realismo crítico y metodológicamente por el dualismo analítico. Para llevar a cabo esta tarea asumimos la perspectiva sistemática de programas multinivelados y la categoría de estrategia de metateorización de tipo preludio de Ritzer.

Palabras clave: morfogénesis, conflacionismo, realismo, dualismo, práctica, social.

\section{Introducción}

La obra de la socióloga británica Margaret Archer contiene uno de los esfuerzos de discusión teórica más profundos de la relación entre estructura y agencia disponibles a la fecha. Dicha relación ocupa el centro gravitacional de sus trabajos, ya sea que hablemos de sus tempranos escritos sobre el sistema educativo 
hasta sus publicaciones más recientes sobre morfogénesis social, modernidad tardía y reflexividad, pasando por su revisión del concepto de hombre y de humanidad y por su permanente reflexión sobre los desafíos europeos y las cambiantes coyunturas de clase. En todos ellos la relación fundamental entre estructura y agencia, ocupa, como en buena parte de la producción disciplinaria británica de posguerra, el centro de la elaboración sociológica y distribuye una gama diversa de originales elaboraciones conceptuales, ya sea en términos críticos, ya sea en términos de innovaciones. En este trabajo nos ocuparemos de uno de los momentos más encumbrados y ricos de la elaboración conceptual desarrollada por Archer para abordar la aludida distinción, a saber, su discusión general del conflacionismo en la sociología.

El inicio de esta discusión puede datarse en 1982, año de publicación del artículo Morphogenesis versus structuration: on combining structure and action y, si bien su pervivencia llega hasta nuestros días, podemos afirmar junto con Lilli Zeuner (Cultural sociology from concern to distance) que el núcleo de los argumentos ya está en el libro Realist social theory. The morphogenetic approach editado en el año 1995. En éste la autora acaba su diagnóstico sobre la sociología conflacionista y reivindica las premisas del realismo crítico y el dualismo analítico, a las que tiene por pilares de una alternativa frente al conflacionismo. A lo largo de esos doce años la autora produjo materiales de notable factura dedicados a esta discusión como el artículo The mith of cultural integration, el libro Culture and agency y el capítulo Taking time to link structure and agency. Cabe resaltar que, en ese marco, inauguró una amplia e intensa polémica con Anthony Giddens y sus teorías de la estructuración y de la dualidad de la estructura, cuya novedad y carácter de work in progress no le impidieron arrojarlas al campo de la sociología conflacionista.

La sociología conflacionista es el tema que nos convoca, ya que entendemos que su análisis y reconstrucción ofrece tres elementos primordiales para acceder de manera cabal al programa de investigación promovido por Margaret Archer, y también para complejizar y enriquecer el debate y el análisis sistemático de la producción teórica en sociología en la posguerra tardía. En la crítica general a la sociología conflacionista apreciamos tres elementos distintivos: primero, un esfuerzo diagnóstico, centrado en identificar y calibrar la presencia y extensión de la falacia de conflación en la disciplina; segundo, un mapeo preciso de las tareas que debe afrontar una sociología alternativa no-conflacionista en materia de pretensiones, posicionamiento hacia el objeto, perspectivas y ubicación de lo social, y tercero, una completa estrategia de metateorización de tipo preludio, centrada en la doble diferenciación del programa de una sociología no-conflacionista de bases sociológicas, pero también interdisciplinarias.

A propósito de esto último, cabe aclarar que tomamos el concepto de metateorización de tipo preludio (en adelante $\mathrm{Mp}$ ) de la propuesta metateórica de George Ritzer (Metatheorizing in sociology), quien propuso integrar los distintos modos de comparación conceptual en sociología, todos los cuales implican el estudio sistemático de teoría sociológica (solo difieren en sus objetivos, las siglas y sus subíndices tienen sentido en inglés). La primera es Mu (Metatheorizing as understanding [comprensión]) e implica que la metateorización es un medio para comprender mejor y más profundamente la teoría existente. Mu concierne al estudio de teorías, teóricos, comunidades de teóricos, tanto como al estudio de los contextos intelectuales y sociales más amplios de las teorías y de los teóricos. La segunda es Mp (Metatheorizing as prelude [preludio]) e implica que la metateorización es un preludio para el desarrollo de teoría, así el estudio de la teoría existente es realizado a efectos de producir una nueva teoría sociológica. La tercera es Mo (Metatheorizing as overarching [transversal]) e implica que la metateorización es una fuente de perspectivas que surcan transversalmente (overarch) la teoría sociológica. Mo es el estudio de teoría orientado al objetivo de producir una perspectiva, que Ritzer denomina metateoría, que cruce transversalmente buena parte, sino toda la teoría sociológica. Al proponer encuadrar la crítica general al 
conflacionismo como estrategia Mp, buscamos observar las operaciones de diagnóstico talladas por Archer como preludios de la postulación de su alternativa programática. Esto es, nos proponemos, guiados por Ritzer, reconstruir el vínculo entre el diagnóstico y la alternativa.

Aclarado este punto, volvemos al programa de Margaret Archer. El núcleo crítico de su diagnóstico sobre la situación de la sociología es el concepto de conflacionismo (conflation). En él Archer aúna a las más heterogéneas teorías sociológicas y culturales, para recriminarles, a todas, incurrir por vías diversas en un mismo defecto: la visión imprecisa e incompleta de la dinámica del mundo social. Este defecto, denominado el conflacionismo o la comisión de falacia de conflación, será el tema de este trabajo y es prudente que ofrezcamos una primera aproximación, un avistaje panorámico del asunto, a fin de introducir al lector en los núcleos de su discusión y que se haga una representación de la magnitud de la misma.

La imputación de conflacionismo se apoya sobre dos supuestos irrenunciables: una ontología estratificada del mundo y un acceso dualista al mundo social. Sin aceptar tales supuestos, el conflacionismo pierde sustento y carece, ya no solo de eficacia crítica, sino de todo sentido, ya que el conflacionismo es un acceso que pierde de vista, tanto la estratificación del mundo como el dualismo de lo social. Repasemos brevemente estos supuestos antes de proseguir.

El primer supuesto del conflacionismo es, como dijimos, una ontología estratificada de la realidad del mundo. En reiteradas ocasiones la autora británica manifestó su asunción de la concepción ontológica estratificada propia del realismo trascendental, luego crítico, de Roy Bhaskar. En dicha ontología, siguiendo a Bhaskar, las cosas (literalmente: things) actualizan o enduran (literalmente: endures) sus estados debido a la acción de mecanismos generativos específicos. Estos mecanismos, y solo éstos, establecen las capacidades, propensiones y poderes de las cosas y, por tanto, delimitan las modalidades de actualización y las posibilidades actualizables de ellas, ofreciendo un modelo de causación contrario a la tesis del determinismo de la regularidad. La acción de tales mecanismos es capaz de formar y estabilizar causalmente estratos de realidad, dotándolos con propiedades emergentes.

Es importante aclarar aquí que los autores del realismo trascendental y crítico, Archer y Bhaskar, usan a lo largo del tiempo y a través de las obras designaciones distintas para el referente "orden de realidad". Así se suceden "estrato" (strata), "dominio" (domain), "nivel" (level), "aspecto" (aspect), "capa" (layer), al tiempo que, en ciertos trabajos, varios de ellos conviven, pero en otros no. Yendo a Archer en Culture and Agency conviven level y aspect, predominando este último, mientras que en $A$ Realist Theory conviven level y strata, con un número de apariciones similar, pero desaparece aspect. Para facilitar la lectura, en este trabajo optamos por emplear los términos "estrato" y "nivel". Reservaremos el primero al campo ontológico, debido a que Archer sigue a Bhaskar en ese terreno, quien priorizó ese término, y haremos lo propio con el segundo para el mundo social, debido a que es el término con mayor permanencia en el derrotero de los escritos de la autora británica. Sin embargo, aclaramos que es solo una decisión operativa, nuestra, y que la tomamos ante esa riquísima proliferación sintagmática, a la que de ninguna manera deseamos eliminar, al contrario, la consideramos relevante y su detallada investigación arrojará resultados sorprendentes.

Estos estratos resultan, por tanto, tan indisociables de los mecanismos generativos que los posibilitan y conforman, como irreductibles a la acción de otros mecanismos de igual tenor, pero de distinta naturaleza. Así visto, un estrato del mundo posee autonomía, una legalidad propia e irreductible, la cual está determinada por las propiedades generativas, emergentes y causales de los mecanismos generativos que 
lo conforman. Debido a esto, todo estrato es ontológicamente irreductible a la acción de otros mecanismos generativos y de otros estratos del mundo. La irreductibilidad ontológica es considerada la condición de posibilidad de la formación de sistemas abiertos, determinados causalmente por la constante interacción entre mecanismos generativos irreductibles, que origina así dinámicas / patrones / estructuras variables dentro del mundo. El conflacionismo será incongruente con esta ontología estratificada puesto que omitirá el principio de irreductibilidad y negará la autonomía de los estratos.

El segundo supuesto del conflacionismo es el acceso dualista a lo social, para ser exactos: la asunción del dualismo analítico como acceso preferible a la realidad del mundo social y que Archer deslinda del dualismo filosófico. Según la socióloga británica, quién cuestionó y corrigió aquí el planteo de Bhaskar sobre lo social, el mundo social es un estrato del mundo, cuyo mecanismo generativo es el interjuego entre dos niveles autónomos, a los que denominará variablemente, ora como "agente/agencia y estructura", ora como "procesos causales" y "principios lógicos", ora como "nivel socio-cultural" (socio-cultural level, o en siglas: S-C L) y "nivel del sistema cultural" (level of cultural system, o CS L), ora como "dominio cultural" (cultural domain) y "dominio estructural"(structural domain). Debido a la asunción de una ontología estratificada, tanto el nivel del agente como el nivel de la estructura poseen propiedades emergentes y son recíprocamente irreductibles. Esto requiere una perspectiva analítica que sostenga la irreductibilidad. Ante ello Archer defiende la preferibilidad del dualismo analítico ya que, a su juicio, es una postura capaz de sostener con firmeza la autonomía de ambos niveles y de observar el carácter morfogenético del interjuego entre ellos. El conflacionismo será inconsistente con el dualismo analítico debido a que accederá a lo social mediante un asalto unilateral y solapará las propiedades de un nivel en las propiedades de otro. Este proceder difumina el acceso dualista al interjuego y vuelve imprecisa y equivoca la distinción de propiedades y características de los respectivos niveles. El resultado final del asalto será indefectiblemente la transposición y supresión de la autonomía de, al menos, uno de los niveles en la autonomía de otro.

Estilo de toda la oración, dice "En este asalto se difumina el acceso dualista, siendo su resultado la supresión de la autonomía de algún/os niveles en en la autonomía de un nivel. ", debe decir "El resultado final del asalto sera indefectiblemente la transposición y supresión de la autonomía de, al menos, uno de los niveles en la autonomía de otro."

Pasemos en limpio la relación del conflacionismo con sus supuestos. Bajo una ontología estratificada y una perspectiva analítica dualista, Archer aísla y caracteriza los defectos generales del conflacionismo, es decir las debilidades que le son inherentes, a saber: el conflacionismo es incongruente con la estratificación e inconsistente e incompleto frente al dualismo.

Ubicados y subrayados semejantes defectos generales, Archer sube la apuesta de su concepto en materia de abstracción. La crítica al reduccionismo es una práctica secular en la investigación social, está presente en los clásicos de la sociología y se puede decir, sin temor a equivocarse, que desde entonces aglutinó una semántica disciplinaria. Hacia los años setenta del siglo XX, ante la ebullición de alternativas ante la expansiva crisis de "las dos sociologías", la crítica al reduccionismo se convirtió en la crítica a los reduccionismos. En el paso del singular al plural se manifiesta una de las principales exploraciones teóricas de salida a dicha crisis: disolver las antinomías en una posición equidistante y ecuánimemente crítica de las dos sociologías. Ni subjetivistas ni objetivistas, ni voluntaristas ni deterministas, ni accionalistas ni estructuralistas. Esta peculiar alternativa fue impulsada titánicamente por un buen número de autores, hoy consagrados, pero noveles en ese entonces, como Coleman, Bourdieu, Schluchter, Giddens, por solo mencionar algunos de los más destacados. Debido parcialmente al interés en esta alternativa, diversos 
analistas como Alexander, Villa y Joas y Knöbl, entre otros, aceptan aunarlos generacionalmente y bautizarlos como "new theoretical movement", o simplemente "new movement". Archer, parte de esa generación, pero para nada parte de ese movimiento, realiza una de las críticas más profundas del reduccionismo sociológico en todas sus variantes, incluida una particular forma de éste originada en la posición del "new movement". La autora británica observa que, en su presunción superadora y sus pretensiones de ecuanimidad crítica, la aspiración de disolver las antinomias generó una nueva práctica reduccionista. Para abrazar a todas las formas de reduccionismo, las de siempre y la novedosa, Archer optó por subir el grado de abstracción del concepto de reduccionismo. Esto la condujo formular el concepto de elisión, un término que provocativamente tomó de Giddens.

To elide ("elidir") significa omitir uno o más fonemas de una palabra durante una alocución oral. En la perspectiva de Archer esto es re-especificado como omitir el poder descriptivo y/o explicativo de un principio, apoyado en la sobrecarga descriptiva o explicativa de otro. Este desbalance es causado por el solapamiento de propiedades de un nivel en las propiedades de otro/s. La elisión en sociología tiene tres variantes principales: la elisión de las propiedades del nivel de la agencia en el nivel de la estructura, la elisión de las propiedades del nivel de la estructura en el nivel de la agencia, y la elisión simultánea de las propiedades de ambos niveles, tanto de la agencia como de la estructura, en un término tercero. Al ejercicio de la elisión, Archer lo denominó elisionismo, y afirmó constituye la forma de conflacionismo predominante en la sociología y el análisis cultural. En consecuencia, la profunda radicación de la elisión en sus prácticas provoca que la disciplina, como tal, sea incongruente ontológicamente e inconsistente e incompleta analíticamente.

Gracias a ello, Archer observa una forma novedosa de elisión característica del "new movement". Incurrir en elisionismo es lo que típicamente han hecho los reduccionismos, cuyas deficiencias están a la vista después de tantas discusiones al respecto. Sin embargo, a juicio de la autora, no sucede lo mismo con la incursión en elisionismo por parte de las nuevas propuestas. Sus deficiencias no están a la vista y ella considera necesario detenerse a exponerlas. El "new movement", según la actual Presidenta de la Pontifica Academia de Ciencias Sociales, también elide. Difiere con los reduccionismos en el modo de hacerlo, pero no en su efectiva hechura. El "new movement" elide, no uno de los niveles del mundo social en el otro, sino los dos. Esto sucede cuando descarga las propiedades de ambos niveles en las propiedades de un término tercero.

La crítica de Archer pone de relieve las graves insuficiencias teóricas del pretendido arreduccionismo del "new movement" debido a que éste, para resolver el estatuto y el rango de la relación entre niveles, insiste en la inseparabilidad de ellos, en la inseparabilidad de partes y personas, en sus palabras: "[T]here is a third form of conflation which does not endorse reductionism at all. There is Central conflation which is areductionist, because it insists upon the inseparability of the 'parts' and the 'people'. In other words, the fallacy of conflation does not depend upon epiphenomenalism, on rendering one level of social reality inert and thus reducible. Epiphenomenalism is not the only way in which the 'parts' or the 'people' are deprived of their emergent, autonomous and causally efficacious properties and powers, and that in consequence their interplay is denied. Any form of conflation has the same consequences [...] This is demonstrated by Central conflation, where elision occurs in the 'middle'. This directional approach, which is reflowering at the moment, interprets neither the 'parts' nor the 'people' as epiphenomena of one another. Indeed, it is precisely their opposition to reduction which is the prime article of faith among modern proponents of Central conflation. Instead, what happens is that autonomy is withheld from both levels because they are held to be mutually constitutive. These theories have been encountered before when examining those who elide culture and agency and structure and agency. As mutually constitutive, 
the two elements cannot be untied and therefore their reciprocal influences cannot be teased out, which is held to be their major defect and one which severely limits their utility in practical social research (...) Their respective approaches to human practices generically preclude one from disengaging the properties and powers of the practitioner from the properties and powers of the environment in which practices are conducted -and yet again this prevents analysis of their interplay. Instead, we are confronted with amalgams of 'practices' which oscillate wildly between voluntarism and determinism, without our being able to specify the conditions under which agents have greater degrees of freedom or, conversely, work under a considerable stringency of constraints" (Archer 2000a:6-7).

Ante el facilismo de esta falsa alternativa, años más tarde, en la introducción de Being human, Archer directamente distinguió entre areduccionismo y antireduccionismo y afirmó, a reglón seguido, que la alternativa realista es firmemente antireduccionista, y solo así podría ser, ya que asume que los niveles no solo son "separables", sino que son irreductibles y autónomos, siendo tarea del realismo social lograr una perspectiva que presente un objeto con tales características y garantice un acceso a él en tales condiciones. Así la crítica al elisionismo abarca la crítica del reduccionismo o elisión unilateral, pero también la crítica a la elisión bilateral de los niveles del mundo social y, por tanto, una explicitación de su incapacidad para observar el interjuego entre ellos. Este grado mayor de abstracción adquirido por los conceptos de elisión y de elisionismo, además de aquilatarlos teóricamente y de extender la cobertura crítica del diagnóstico de Archer más allá del concepto de reduccionismo, ofrece un pequeño, pero interesante, conjunto de lecciones, primero, el reduccionismo no agota el elisionismo, o inversamente el conflacionismo no depende del epifenomenalismo; segundo, tanto el reduccionismo como el arreduccionismo son elisionistas; tercero, la postulación de un tercer término o tertius no garantiza per se ninguna salida del elisionismo, además del atributo de "terceridad" el término requeriría premisas antireduccionistas; cuarto, la posición arreduccionista, con tertius o sin él, es insuficiente para salir del conflacionismo, la alternativa integral es el anticonflacionismo, y quinto, la opción anticonflacionista tiene afinidades electivas con el dualismo y el realismo.

Ganada la abstracción para el concepto y aprehendido el "new movement", Archer acomete la tarea de caracterizar lógicamente a la elisión sociológica. El resultado es la afirmación de que la elisión opera una falacia, a la que denomina falacia conflacionista, en la investigación sociológica. La característica distintiva de esta falacia es describir y explicar lo social hipostasiando el primado de un nivel por sobre otro/s. Esto genera una asimetría sociológica entre las propiedades simétricas de los niveles, establece una relación de premisas mayores y menores entre premisas iguales y genera predicados incongruentes e incompletos. La incursión en esta falacia malogra por fuerza los rendimientos científicos de la sociología, pues sus pretendidas descripciones y explicaciones no son más que predicados que cargan con esos defectos y, por tanto, prescinden injustificadamente de al menos uno de sus niveles. Así, basada en la falacia conflacionista, la sociología es incapaz de modelar, acceder y caracterizar científicamente la capacidad morfogenética del interjuego entre estos y sus predicados sobre el mundo social son "mistificaciones".

Gracias a esta panorámica, se entrevé ya que los conceptos de conflación y elisión, así como su remisión recíproca, fueron forjados bajo el fuego de una encendida discusión sobre la cientificidad de la sociología. Ella templó la fuerza crítica del diagnóstico integral de Archer sobre la sociología conflacionista y la necesidad de construir una alternativa congruente con una ontología estratificada, afianzada en una epistemología realista y consistente con una metodología analíticamente dualista. En esta línea, la autora subrayó que en el caso del conflacionismo central, la elisión y la conflación son areduccionistas, mientras que, en la opción realista seguida por ella también, es antireduccionista. El matiz es interesante, porque los primeros siguen el método de prescindir de la reducción epifenoménica, mientras que los segundos se 
oponen y así logran observar que los areduccionistas incurren en conflación cuando exploran las formas de la dualidad, pagando el precio de suprimir la autonomía de los niveles bajo estudio. Bajo este esquema de premisas la autora británica identificó tres modos o métodos conflacionistas: conflacionismo descendente, conflacionismo ascendente y conflacionismo central. Luego estableceremos los nexos entre este diagnóstico y el delineamiento de la alternativa de la teoría social morfogenética. Nuestro plan es reconstruir y analizar cada uno de estos aspectos, y finalmente, en la última sección del trabajo, sintetizaremos los resultados alcanzados y extraeremos las conclusiones sistemáticas del caso.

\section{El origen mítico de la sociología conflacionista: integración cultural y elisión}

Archer asume que el concepto de cultura no ocupa un lugar claro en la sociología porque es descriptivamente vago y explicativamente antojadizo. La vaguedad ha alcanzado un punto tal que nadie puede decir sociológicamente ni qué es la cultura ni qué hace. Según la autora, esta situación crítica tiene su origen en la asociación fuerte entre el concepto sociológico de cultura y el concepto originalmente antropológico de integración cultural. Debido a dicha asociación las graves deficiencias de este último son trasladadas al núcleo conceptual del primero, de manera que el mito de la integración cultural toma la forma del concepto de cultura y lo puebla de falacias y dificultades, que hoy podríamos calificar como obstáculo epistemológico.

El carácter mítico de la integración cultural, Archer lo retoma explícitamente de las ideas de Etzioni. El eje crítico del planteo es la asunción aproblemática de un alto grado de consistencia en las interpretaciones producidas por las unidades societales. Según Archer, esta asunción impone el principio de que la organización social es compacta y coherente. Semejante asunción tiene su origen, siguiendo a la socióloga británica, en el programa de investigación de la antropología civilizatoria, cuyas ambiciosas pretensiones descriptivas lo llevaron a hacer propio, a través de un intenso diálogo con el historicismo alemán, el supuesto de que la cultura sería un todo integrado. En el seno de dicho programa antropológico, el supuesto fue erigido como principio unitario de la organización socio-cultural, siendo operacionalizado en cuanto tal. De esta manera fueron asentadas en él las bases teóricas que permitirían caracterizar y describir la "unidad cultural" de entidades del tipo "todos colectivos" (collective wholes). El mito surge cuando esa descripción es elevada al rango de arquetipo de la(s) cultura(s), convirtiéndose toda cultura en una "perfectly woven and all-enmeshing web" (Archer 1996:2). Tal mito llega a nuestros días bajo la figura de que la cultura sería un sistema perfectamente integrado, siendo cada uno de sus elementos interdependientes de todos los otros. Según Archer, bajo el primado del mito de la integración cultural, no es posible la emergencia de ninguna sociología de la cultura.

El mito reposa en dos operaciones fundamentales: 1) atribuir a los patrones culturales las propiedades de unidad subyacente y de coherencia fundamental, de manera que la consistencia del sistema social es homóloga a la coherencia lógica, 2) descargar las propiedades de (1) en un concepto de acción uniforme, debido a lo cual ella, y solo ella, produce homogeneidad social, de manera que la razón del éxito integrador del sistema social descansa sobre un consenso causal. De acuerdo con esto, "una comunidad de sentidos compartidos (shared meanings)" (Archer 1985:336) elide al primer término (comunidad) en el segundo (sentidos). Así, y solo así, un sistema societal es dotado con la (curiosa) propiedad de consistencia interna alta, a la que denominará (y mistificará como) integración cultural, volviéndose la coherencia lógica de las ideas el núcleo sociológico de la consistencia social de los sistemas sociales.

El gran problema de este mito para Archer es que confunde dos elementos con propiedades lógicas y sociológicas bien distintas, como lo son la agencia por un lado y las estructuras por otro lado. Este es el 
núcleo confuso, originado en la antropología civilizatoria y transmitido y extendido en la sociología, de una concepción de cultura basada en el principio de integración cultural. Según Archer, toda sociología que incorpore este núcleo conceptual no sería capaz de apreciar el papel crucial desempeñado por el interjuego a través del tiempo (interplay over time) entre los dos niveles, míticamente confundidos en la dinámica cultural. Tal confusión es inevitable en el marco de referencia del mito, porque es propio de él concebir la unidad integrada mediante la confusión de niveles distintos. Por tanto, al incluirlo en la elaboración conceptual, en nombre del acceso al núcleo unitario e integrador del sistema social, elide (elides) forzosamente la autonomía de al menos uno de los dos niveles en el otro y así se enceguece ante el interjuego entre ellos.

Esta elisión de niveles es bautizada como falacia de conflación e indica el desvanecimiento de las propiedades lógicas y sociológicas de un nivel en las propiedades lógicas y sociológicas de otro. Así, la falacia confunde lo lógico y lo causal, es decir, confunde las propiedades lógicas y los procesos causales. Archer se encarga de enfatizar que la conflación consiste tanto en suprimir la autonomía, ora de las propiedades lógicas, ora de las relaciones causales, como también en denegar su interjuego. Lo propio de la conflación es producir ambas consecuencias. De manera que la reducción epifenoménica, o epifenomenalismo, se debe considerar solo un caso particular del error genérico de conflación. La autora conduce el argumento hasta el punto de afirmar que hay maneras noepifenomenalistas de conflacionar. En ellas la conflación de lo lógico y de lo causal sucede sin reducir ni lo lógico a lo causal ni lo causal a lo lógico, sino elidiendo ambos en un término tercero pretendidamente situado en el "medio" de lo causal y de lo lógico. Mascareño sintetizó con claridad el planteo: "Por conflacionismo entiende [Archer] la anulación del carácter autónomo de la acción, de la estructura o de ambas, es decir, la integración -y con ello pérdida o fusión- de la autonomía de la acción en la estructura, de la autonomía de la estructura en la acción, o de ambas en un tercer elemento" (Mascareño 2008:224).

La falacia de la conflación es el corazón de toda la sociología conflacionista y se pone a disposición bajo dos métodos principales. En el primer método a las propiedades del nivel elidido se las considera derivadas, segundas, o premisas menores de las propiedades del otro nivel al que se tiene por fundamental, primero, o premisa mayor. A las propiedades del nivel elidido se las considera epifenómenos y al nivel que las absorbe se lo establece como sociológicamente fundamental. Este método, y solo él, genera una asimetría entre las propiedades de uno y otro nivel, estableciendo entre ellos una jerarquía entre ellos. A este método de conflación Archer lo denomina conflación epifenoménica y lo desagrega, a su vez, en dos modalidades: la primera es el epifenomenalismo desde arriba, o conflación descendente, en este caso las propiedades del nivel de la elaboración cultural son elididas en las propiedades de las condiciones culturales, o dicho con más abstractamente: lo causal es elidido en lo lógico. La segunda es el epifenomenalismo desde abajo, o conflación ascendente, donde las propiedades del nivel de las condiciones son elididas en las propiedades de la elaboración cultural, o en un lenguaje analítico: los principios lógicos son elididos en los procesos causales.

En el segundo método, la elisión es operada a partir de la incorporación de un tercer término, que pretende ser colocado en el justo punto medio de ambos niveles. En este caso las propiedades de los dos niveles del interjuego, y el interjuego mismo, son elididas en las propiedades lógicas y sociológicas del nuevo término. Este modelo tiene una sola modalidad de conflación a la que Archer denomina conflación central, por su pretendida aspiración de ubicarse en el justo punto medio, en el centro, de los niveles para evitar toda reducción. 
Obtenemos así un esquema con tres versiones de conflación posibles: conflación descendente, conflación ascendente y conflación central. En las secciones que siguen analizaremos en detalle cada caso.

\section{Conflación descendente (downwards conflation)}

Se entiende por conflación descendente a la elisión de las propiedades del nivel sociocultural en las propiedades del nivel del sistema cultural. Dicho de otra manera, consiste en la conflación de las propiedades lógicas y sociológicas de la agencia en las propiedades lógicas y sociológicas de las estructuras. $\mathrm{O}$ aún de otra manera: conflación descendente es la elisión de los procesos y/o las relaciones causales en las propiedades causales y la asunción de la deductibilidad de aquéllas respecto de éstas. Años más tarde, con la asunción definitiva del realismo crítico, Archer vinculará este tipo de conflación con el colectivismo. La autora insistirá en la convicción metodológica de éste, y dirá que en ella hay una conflación de la acción en el positivismo y en la metodología de las "propiedades colectivas", cuya influencia será considerada por esta tradición como determinante sobre la regularidad de los eventos, pero fracasará en la tarea de establecer una ontología social alternativa a la del individuo.

Si bien Archer opta mayormente por el lenguaje analítico, en ocasiones puntuales ilustra sus puntos con ejemplos. A continuación, reproducimos uno con el ilustra la posición colectivista y su diferencia con la individualista, sobre cuya base objetará su incursión en conflación descendente: "It also follows that if the 'social structure' is only an aggregate, then 'the group' becomes synonymous with 'the social' to the Individualist. Here, the Collectivist queries whether in studying society we are, can, and should be, confined to the study of 'groups'. When we examine kinship structure, for example, we are not just investigating how that 'group' does inter-marry, transmit property, have particular obligations towards specific others and so on, but what rules govern their inter-marriage etc. Comparison of kinship structures is to compare different rules not different groups, for the rules regulate what the members do. Certainly, the continued salience of any rule depends on people continuing to adhere to it (this is merely a statement of activity-dependence) but their adherence is not what makes the rule, otherwise rules just become descriptions of what people do and have no regulatory or constitutive function. The identical point can be made about all other social or cultural institutions" (Archer 1995:43).

El punto de referencia de este conflacionismo es el prius dado a la coherencia cultural del sistema social y la construcción del objeto de acuerdo con la capacidad ordenadora de la consistencia lógica. Esto es, los principios de los patrones culturales ordenan consistentemente las relaciones de las acciones, conformando un sistema social coherente y regular. Aquel prius se manifiesta en la consagración de la coherencia cultural como el problema rector que ordena la constelación problemática y sus ejes de trabajo, especialmente en materia de claves, códigos y cohesión, donde las prácticas no son consideradas más que como accidentes o circunstancias accesorias de la -mítica-integración cultural, que es garantizada lógicamente por los principios del "nivel del sistema cultural", o "desde arriba" de aquéllas. En este punto Archer introduce una interesante distinción de tres versiones de conflacionismo descendente en correspondencia con el eje que predomine para alcanzar la mítica integración cultural. Así la autora distingue, primero, la tesis epifenómenica de la sociología de Sorokin en diálogo con la antropología civilizatoria donde predomina la coherencia; segundo, la tesis normativista del funcionalismo donde predominan las claves o "keynotes", y tercero, la tesis simbólica del estructuralismo donde predominan los códigos. Asimismo, incorporará en ella algunas posiciones neomarxistas como la de Althousser, de quien subrayará su remarcado interés por el funcionalismo de Parsons. Más tarde la autora incluirá al "determinismo tecnológico" y al "economicismo" (Archer 1995:81) entre las corrientes que incurren en este método falaz. 
Archer ampliará sus cuestionamientos a este método al analizar su segmentación del tiempo. Según la autora británica, los supuestos conflacionistas descendentes conducen a segmentar unidades temporales dentro del tiempo social en las cuales se puede observar únicamente la preminencia de la estructura sobre los agentes. Estos segmentos son recortados según el molde de la estructura, de manera que este conflacionismo se limita a verificar en dichos períodos la preexistencia de las condiciones estructurales respecto de los agentes y de la elaboración estructural. Gracias a esta segmentación del tiempo social, verificará a posteriori la perpetración en el presente de los agentes y su interacción de las estructuras pasadas. Esta segmentación implica, primero, despojar a los agentes y sus interacciones de propiedades sociales emergentes; segundo, excluir del espectro temporal la elaboración estructural; tercero, eliminar la dimensión del "futuro", porque el esquema condiciones/perpetración no tiene nada más que observar una vez verificada la puesta en acto de tendencias ya presentes en las condiciones estructurales al momento de la interacción; cuarto, reduce el "presente" a la corroboración de la impresión de la estructura sobre el agente (impress upon), y quinto, suprime del análisis el tiempo de la elaboración estructural presupuesta por las condiciones estructurales, denominado T1, razón por la cual elimina su origen social.

\section{Conflacionismo ascendente (upwards conflation)}

Se entiende por conflación ascendente a la elisión de las propiedades del nivel del sistema cultural en las propiedades del nivel socio-cultural. Dicho de otra manera, consiste en la conflación de las propiedades lógicas y sociológicas de las estructuras en las propiedades causales y sociológicas de agencias, actores o grupos de ellos. $\mathrm{O}$ aún de otra manera: conflación ascendente es la elisión de las propiedades lógicas en los procesos y/o las relaciones causales y la asunción de la deducción de aquéllas respecto de éstas. Años más tarde, con la asunción definitiva del realismo crítico, Archer vinculará este tipo de conflación con el individualismo. La autora insistirá en el compromiso ontológico del individualismo y dirá que en él hay una conflación de lo estructural en una ontología empiricista del individuo, lo cual exige completar la demostración del carácter epifenómenico de las estructuras.

El punto de referencia de este conflacionismo es el prius dado a la uniformidad socio-cultural del sistema social y la construcción del objeto de acuerdo con el poder causal de la formación de consensos. Esto es, los principios de la interacción ordenan consensualmente los particulares como generales y así manipulan las propiedades lógicas de los patrones culturales, conformando un sistema social consistente. Aquel prius se manifiesta en la consagración de la uniformidad como el problema rector que ordena la constelación problemática y sus ejes de trabajo, especialmente en materia de consenso y manipulación. Al momento de organizar los materiales y las teorías que, a juicio de Archer, hacen sociología y análisis cultural de esta manera, somos sorprendidos por la autora británica quien, en un primer momento, se concentró casi exclusivamente, al menos en lo relativo al análisis cultural, en el "marxismo occidental", más allá de inclusiones express como las que convida a Comte y a Mannheim. Desde los jóvenes Marx y Engels hasta Habermas, pasando por algunos "leninistas" -que no son mencionados-, por Gramsci y por representantes de la primera escuela de Frankfurt, a todos Archer los aglutina en la tesis del "manipulated consensus" de la ideología, que sería un modo de alcanzar el mito de la cohesión cultural, pero no a priori o "lógicamente" desde "las claves, los códigos y la cohesión", sino desde los procesos causales originados en las practicas inscriptas en el "nivel socio-cultural", y únicamente desde ellas, o sea "desde abajo". Igualando el procedimiento hecho con el conflacionismo descendente, Archer distingue tres versiones de este método: la tesis ideológica del marxismo clásico forjada, a su entender, en La ideología alemana de Marx y Engels, y luego subdivide en dos a lo que se permite denominar como "neomarxismo": la tesis del 
instrumentalismo representada paradigmáticamente por Gramsci y la tesis de la conciencia tecnocrática defendida por la Teoría Crítica in toto, siendo su representante más acabado Habermas.

Sin ejemplificar puntualmente esta modalidad de conflación, Archer analiza y comenta críticamente -no sin procurar una recuperación parcial de Marx para su programa- las bases conceptuales y los modos de interpretación cultural y sociológica de esta posición: "Marx in admitting to (logical) pluralism amongst the dominant ideas then tried to deprive them of any independent downward influence by signifying upward (causal) pressures which unify them. In the process he was in effect compelled to concede the analytical separateness of the 'logical' and the 'causal' (since there was a two-way interplay between them). Effectively this then imposed a task on neo-Marxists, namely to specify the actual mechanisms by which the Socio-Cultural level socially (not ontologically) nullifies part of the Cultural System (that is, when the latter threatens the former). Dominant ideology theorists have turned their backs on this task by simply denying pluralism and insisting on a single dominant culture. As upward conflationists they have made the logical depend exclusively and unproblematically on the causal, thus rendering the Cultural System as epiphenomenal as was the Socio-Cultural level to downward conflationists" (Archer 1996:50).

Recién varios años más tarde, caído ya el Muro de Berlín, y gracias a un estrechamiento de la asociación de este modo de conflacionismo con las premisas fundamentales del individualismo, la actual presidenta de la Pontificia Academia de Ciencias Sociales incorporó a ciertas tradiciones accionalistas y/o individualistas de la sociología, en particular a la que denominó neophenomenological schooly, sobre todo, al methodological individualism. Operación que repitió con la rational choice algunos años después. Quien fuera presidente de la ISA durante los años 1986-90 ejemplificó a grandes rasgos la variante individualista de la siguiente manera: "[T]o upward conflationists it is always a major descriptive error to treat structural properties as having the ontological status of facts rather than facticity, and it is equally erroneous to allow them to figure in explanatory statements as external conditioners of action. Thus, for instance, the neophenomenological school asserts the primacy of agency by reducing the structural context of action to a series of inter subjectively negotiated constructs. However, the basic charter of all versions of upward conflation, of which interpretative sociology is only one variant, is methodological individualism. Its prime injunction is to view so-called structural properties as reducible to the effects of other actors, which are in their turn always recoverable by agency. Essentially, structure becomes epiphenomenal in classic statements of methodological individualism because the social context is defined as made up of nothing more than other people. For this strategy of 'personalization' to work in social theory, its protagonists have to show that all structural properties (every aspect of the social environment), which figure in explanations, refer to nothing more than the activities and attitudes of other people. Thus, the argument goes, since society is made up of people there is nothing in the environment (although it may appear to be non-people) which people in turn cannot change, leaving aside its physical components. Hence, to Watkins, the 'central assumption of the individualist position -an assumption which is admittedly counterfactual and metaphysical- is that no social tendency exists which could not be altered if the individual concerned both wanted to alter it and possessed the appropriate information'" (Archer 1995:8485).

Análogamente a lo hecho con el conflacionismo descendente, Archer ampliará sus cuestionamientos analizando su segmentación del tiempo. Según la autora británica los supuestos conflacionistas ascendentes conducen a segmentar unidades temporales dentro del tiempo social donde únicamente es observable la preminencia de los agentes sobre la estructura. El segmento resultante adquiere, naturalmente, su silueta del molde de los agentes, no de la estructura ni del interjuego. Esta segmentación temporal, denominada por la autora T3, se limita a verificar en los periodos seleccionados la 
contemporaneidad de los agentes y de las interacciones con que estos se condicionan y constriñen de manera recíproca cuya duración no se prolonga más allá de los “inocentes" productos de los agentes concernidos. Esto implica, primero, despojar a las condiciones de propiedades sociales emergentes y estrechar el sentido de aquéllas a meras "disposiciones para la acción"; segundo, discontinuar la acción respecto de los condicionamientos históricos y "autonomizar" el tiempo de la acción hasta el punto de hacer al agente responsable de su propio origen; tercero, desacopla la dimensión del "futuro" respecto del presente porque el esquema disposición/acción no tiene nada más que observar una vez agotada la temporalidad de las acciones concernidas; cuarto, reduce el "presente" a la "autonomía temporal de la acción", dejando fuera del espectro temporal al condicionamiento estructural y a las consecuencias de la interacción, sean éstas de conservación y/o de influencia, y quinto, suprime del análisis el tiempo de la elaboración estructural presupuesta por las condiciones estructurales, denominado T4, razón por la cual es ciega ante el inicio de relaciones causales.

\section{Conflacionismo central (central conflation)}

Se entiende por conflación central a la elisión simultánea de las propiedades del nivel del sistema cultural y de las propiedades del nivel socio-cultural en las propiedades de un término tercero situado entremedio de ambos. Dicho de otra manera, consiste en la conflación de las propiedades lógicas, causales y sociológicas de las estructuras tanto como de las agencias, actores o grupos de ellos en las propiedades de un término sociológico tercero, que no es ni estructura ni agente/actor. O aún de otra manera: conflación central es la elisión de las propiedades lógicas y de procesos y/o las relaciones causales en un término pretendido mutua y simultáneamente constitutivo de ambas.

El punto de referencia de este conflacionismo es el prius dado a la constitución mutua de estructura y agencia y en la construcción del objeto de acuerdo con la posibilidad y la capacidad de conexión (linking) y de intermediación de y entre estos niveles. Esto es, los principios de la constitución mutual ordenan simultáneamente las determinaciones recíprocamente referidas de los niveles, conformando una misma realidad social de dos caras. Aquel prius se manifiesta en la consagración de la dualidad de la estructura como el problema rector que ordena la constelación problemática y sus ejes de trabajo.

Archer ampliará sus cuestionamientos a este método conflacionista al incluir un análisis de su segmentación del tiempo social. Según la autora británica los supuestos conflacionistas centrales conducen a recortar unidades temporales únicamente donde haya conjunción entre estructura y agente, es decir segmenta instantes, unidades acotadísimas de tiempo. En sus propias palabras: "Thus, structural properties (defined reductively as rules and resources) are held to be outside time, having a 'virtual existence' only when instantiated by actors. In exact parallel, when actors produce social practices they necessarily draw upon rules and resources and thus inevitably invoke the whole matrix of structural properties at that instant" (Archer 1995:87).

Esta segmentación temporal observada por la autora, a la que denomina T2-T3, se limita a observar el interjuego entre estructura y agencia en periodos de tiempo muy acotados, debido a que ambos términos son presupuestos en excesiva proximidad, una cercanía que le mereció a Archer el mote de "methodological bracketing", desconociendo que estructura y agencia trabajan en intervalos de tiempo diferentes. En este punto la autora explicita que su tesis es abiertamente polémica con las pretensiones de la teoría de la estructuración de Giddens respecto del objetivo de incorporar integralmente al tiempo en la teoría social. Al respecto del "metodological bracketing" la autora sugirió que "[it] has produced a pendular swing between contradictory images of chronic recursiveness and total transformation" (Archer 
1995:88). Más allá de la aguda ironía, esta crítica a Giddens implica cuestionar, primero, la aproximación de los términos hasta acabar por cohesionarlos y considerarlos co-terminous in time, es decir, hasta asumirlos como dos lados de una y la misma realidad social; segundo, la obturación, e incluso el impedimento del examen lógico de las relaciones temporales entre estructura y agencia; tercero, el desacoplamiento de la dimensión del "futuro" respecto del presente porque desconoce la estructuración a lo largo del tiempo (over the time); cuarto, la reducción del "presente" al "momento" o "instante", en cuya acotada unidad temporal son descargadas simultáneamente propiedades temporales de la estructura y también de los agentes, marginando la temporalidad de la estructura como "virtualidad" y privando a los agentes de la propiedad de selectividad estructural; quinto, la incapacidad para distinguir las relaciones temporales entre estructura y agencia en los puntos T1 y T4, esto se debe a que la teoría de la estructuración es incapaz de distinguir temporalmente entre condicionamientos (T1) y elaboraciones estructurales (T4), dicha incapacidad condujo a dicha teoría, según Archer, a desdiferenciar, pero a la vez a reclamar por el "momento" de "replicación" y/o de "fase crítica"; de manera que en sexto y último lugar, al ser incapaz de distinguir T1 de T4, es también incapaz de observar el interjuego de los términos durante periodos de tiempo largos o prolongados y de distinguir temporalmente, en consecuencia, relaciones lógicas de procesos causales.

A diferencia de lo que ocurre con las otras dos versiones del conflacionismo, Archer acostumbra ilustrar abundantemente esta posición. A continuación, reproducimos un interesante ejemplo, comentado críticamente por la autora: "[T]he Elisionists' remaining way out is to conceptualize such educational restrictions as the unintended consequences of social practices. Thus, Giddens himself gives the example of a poverty cycle where maternal deprivation -poor schooling-low paid employment-maternal deprivation operate as a homoeostatic loop coordinated and controlled exclusively through the unintended consequences of day-to-day social activity. What this does not explain is why some groups enter the loop in the first place, for it is not their practices which set their life chances, determine the definition of instruction, the linkages between educational and occupational opportunity etc. Nor can any of their practices extricate them from the loop without overcoming stringent constraints whose differential distribution again begs the question of why and how they are differentially distributed in society. Any attempt to dispose of these questions by simply invoking the unacknowledged conditions of action, which is part of the Elisionist equation of social structure with practical knowledge, finds itself in the same cul de sac. Victims of educational discrimination are not victimized by their lack of 'discursive penetration' of the situation in which they find themselves. We could endow them with all the findings of educational sociology without changing the fact that their situation places objective limitations on the resources at their disposal and the rules they are able to follow. To know that public schools convey educational advantages which inner city comprehensives do not is only useful to those with the means to turn their knowledge into practice. Thus, when 'structural properties' are defined as 'rules and resources' in order to construe them as co-extensive with 'social practices' there is always a remainder which cannot be accommodated. The ontology of praxis constantly comes up against an interface with another level of social reality whose features cannot be construed as practices themselves, their unacknowledged conditions or unintended consequences" (Archer 1995:116).

El remate de la crítica de Archer al elisionismo es negarle la inscripción en cualquier tradición sociológica. Al respecto señala dos elementos, por un lado, la antropología civilizatoria se habría enfrentado a estos supuestos y los habría caracterizado como un problema, no como una solución y, por otro lado, la teoría social británica habría vuelto a este planteo, pero los habría caracterizados como el punto de salida a la crisis de las sociologías reduccionistas y estaría enarbolando la constitución mutual y la conjunción como resolución definitiva de aquélla. Esta posición, a decir de la autora, no constituiría por sí misma ninguna 
tradición, sino que estaría aún -fines de la década del ochenta- en proceso de cristalización, e identifica dos autores comprometidos con ella: Anthony Giddens y Zygmunt Bauman. Años más tarde, la autora incorporará a Pierre Bourdieu en esa lista, cuyo concepto de práctica cuestionará en los términos precedentes, concluyendo finalmente que se trata de otro posicionamiento de tipo elisionista. En contra del cuál la autora reafirmará su concepción analíticamente dualista (no dual), epistemológicamente realista-crítica y ontológicamente estratificada de las prácticas, a los efectos de reivindicar el concepto de práctica e impedir que este tan importante concepto quede embebido de conflacionismo central. Si bien es cierto que algunos autores han revisado este profundo cuestionamiento de Archer a la posición de Bourdieu, como por ejemplo Elder-Vass, Fleetwood, Aguilar, o más recientemente Aedo, sin embargo, más allá de la excitación que puedan provocar exploraciones de este tipo, la autora se ha mostrado reacia a integrar posiciones. A propósito, hasta el día de hoy, Archer conserva su postura de no aunar a los elisionistas, Bourdieu incluido, en una tradición.

\section{La postulación de una alternativa programática desmistificadora y no-conflacionista}

Desde un punto de vista sistemático, con su crítica de la sociología conflacionista, Archer establece el punto de partida de una sociología alternativa de carácter no-conflacionista. En su caso, durante los doce años en los que elaboró, amplió y precisó su crítica al conflacionismo, exploró en simultáneo diversas alternativas frente a él. En esta línea sopesó los aportes del dualismo analítico, del enfoque morfogenético de la estructura, como así también de la teoría de sistemas sociales de Buckley, realizando además una notable recuperación (y reivindicación) de la distinción entre integración social e integración sistémica de Lockwood. El punto neurálgico de estos diálogos es, a nuestro entender, el interés de la autora por la ontología y la epistemología del realismo, primero trascendental, luego crítico de Roy Bhaskar.

Este complejo doble movimiento de discusión diagnóstica, por un parte, y de desarrollo de alternativas no-conflacionistas, por otra parte, desembocó hacia 1995 en la elaboración conceptual de una íntegra red de elementos teóricos generales (ontológico, epistemológico y metodológico) y de elementos teóricos disciplinarios (unidad de análisis, unidad de orden, unidad de cambio) capaz de oficiar de fundamento operativo de un programa de investigación alternativo en sociología: el denominado realismo crítico morfogenético.

Esta posición procura establecer un esquema de elementos generales fuertemente orientado a las cosas mismas, como gustan decir además de los fenomenólogos, los realistas. Esta orientación es, sin embargo, anti-empirista por antonomasia y explora la elaboración conceptual de tales cosas y de los accesos científicos a ellas sobre la base de la paradoja, los mecanismos generativos, la dinámica de los procesos y la imputación de propiedades autónomas a los distintos estratos de cosas, agrupadas por mecanismos generativos, y no por esencias, cualidades o sustancias. No se trata de asumir que las cosas simplemente "están" o "son", sino que las generan mecanismos. En esa línea la recepción de la ontología y de la epistemología realista y estratificada es crucial. A cuenta de ello Archer, al desarrollar una posición metodológica con preminencia del dualismo, hace su aporte a la discusión realista general. La autora enfatiza la autonomía de los niveles emergentes y la necesidad de acceder a ellos analítica, no sintéticamente. Esta posición se pretende extensiva de las premisas estratificadas y realistas, y se aloja a los agentes y a las estructuras en niveles estratificadamente distintos. La postulada existencia de un interjuego entre niveles reposa, es informada y regida por esa concepción general, y se pretende garantizar la no-eliminación de tales premisas. 
Visto desde un punto de vista estrictamente disciplinario ahora, latamente sociológico, el aludido interjuego y su infraestructura analíticamente dualista es aunado por Archer en un único concepto de "lo social": la variación interdependiente entre las relaciones lógicas y las causales en un primer momento, luego la relación (relationship) entre el nivel del "agente" y el de la "estructura" (Archer 1995:133) y finalmente el ciclo morfogenético que será doble (morfogénesis/morfostasis) y triple (persons, agents, actors). Este denodado esfuerzo de unidad procesual es una marca distintiva de la postura de la autora británica. En esta concepción de socialidad estratificada y dualista, la unidad de lo social y, por extensión, la unidad de análisis de la sociología, adopta la forma de ese mecanismo generativo. En esta red de elementos disciplinarios el ciclo morfogenético no es ni el punto ni el momento de desdiferenciación de agente y estructura, sino el mecanismo generativo de la elaboración estructural en el que ambos niveles con plena autonomía concurren a través del tiempo. Archer coloca el acento de su concepción de socialidad sobre el proceso, no sobre la estructura, y ahí, y solo ahí, es donde adquiere unidad social. La práctica social abandona tras de sí las propiedades acontecimentales y evanescentes que los planteos elisionistas gustan atribuirle y adquiere las ropas de un interjuego procesual, con propiedades temporales unitarias, pero longitudinales. En este sentido, la puesta en perspectiva morfogenética de la unidad de los procesos y de sus propiedades emergentes es solo un paso más del planteo. Su adopción por parte de Archer no resulta ni forzada ni antodajiza, sino participe de la fundamentación de un planteo centrado en la unidad del proceso.

La originalidad de la exploración acometida por Archer fuerza la exposición de ejemplos de la flamante posición por ella propiciada. Este es el lugar ocupado por el relativamente célebre "Castro's example" (Archer 1995:77), que hace referencia al proceso de alfabetización masiva encarado en tiempo record por la revolución cubana en los años sesenta y analiza algunos escenarios posibles a través de la observación, centrada en el concepto de ciclo morfogéntico y apoyada en la distinción morfogénesis/morfostasis, de los resultados parciales alcanzados cada vez por la política de alfabetización masiva y su reintroducción en la política de desalfabetización.

Si avanzamos sobre estas premisas desde un punto de vista arquitectónico, podemos desagregar cada una de las redes. A saber, la red de elementos teóricos generales implica un componente ontológico centrado en una concepción emergentista y estratificada del mundo, un componente epistemológico basado en el realismo crítico y un componente metodológico centrado en el dualismo analítico. Asimismo, la red de elementos disciplinarios tiene en la práctica social la unidad de análisis, en el interjuego entre estructura y agencia la unidad de orden, y en la morfogénesis la unidad de cambio.

De esta manera, los componentes del fundamento operativo general deslindan una posición que abraza, en cada uno de sus pasos, la mejor o la más fuerte de las opciones anticonflacionistas que tenga a disposición, y en caso de no hallarla, tal es la línea que se da para elaborarla conceptualmente de manera original. Las pretensiones de universalidad del programa son meridianas. No se propone abordar ni un campo especial ni una zona geográfica, sino el conjunto de fenómenos sociales. Adopta hacia el objeto una actitud práctica, se sitúa en su mismo campo y, junto con la tarea de conocerlo en sus mecanismos generativos, asume la tarea de participar de sus cambios mediante "Practical Social Theory" informadas por la perspectiva general de la ontología social metateórica (meta-theoretical social ontology) del realismo crítico y el abordaje metodológico (metodological approach) de la morfogénesis. La ubicación de lo social es la de un mecanismo generativo, denominado ciclo morfogenético, que procesualiza la infraestructura dualista de los dos niveles del mundo social. Tales son los puntales del programa alternativo que Margaret Archer forjó bajo el fuego del no- o, directamente, anti-conflacionismo. 


\section{Conclusiones. De la crítica al conflacionismo al realismo crítico morfogenético}

A lo largo de este trabajo nos dedicamos a reconstruir los elementos salientes de la crítica a la sociología conflacionista planteada por Margaret Archer, entendiéndola como la principal elaboración diagnóstica propuesta por la prestigiosa autora británica de cara a la actualidad de la sociología. Entendimos también que dicho diagnóstico no es ningún elemento aislado, sino que ofrece un mapeo de focos críticos ante los cuales elabora lineamientos alternativos de salida de tal escenario. En esa complementación contrastiva entre diagnóstico y alternativas observamos la unidad de una estrategia de metateorización de tipo preludio propia del realismo crítico morfogenético de Archer.

A continuación, en la tabla 1, sintetizamos los resultados alcanzados y los organizamos según los ejes relevados por la autora. Cada columna se corresponde con un eje diagnosticado y con la proposición de una alternativa, mientras que cada fila se corresponde con una puntualización de las características problemáticas y sus diversas raíces y operaciones específicas. Esto redunda en una constelación de ejes de diagnóstico y alternativa, las primeras tres filas agrupan las fuentes primordiales de los focos críticos, mientras que la cuarta puntualiza la posición alternativa al conflacionismo delineada y fundamentada por Archer.

Tabla 1. Síntesis de resultados alcanzados (elaboración propia)

\begin{tabular}{|c|c|c|c|c|}
\hline & Colectivismo & Individualismo & Elisionismo & Morfogénesis \\
\hline Conflación & Descendente & Ascendente & Central & -- \\
\hline $\begin{array}{l}\text { Relación entre } \\
\text { niveles }\end{array}$ & Anti reduccionismo & Reduccionismo & Arreduccionsmo & $\begin{array}{l}\text { No reduccionismo o } \\
\text { anti reduccionismo }\end{array}$ \\
\hline Prius & Estructura & Agencia & Mutual constitution & Interplay over time \\
\hline $\begin{array}{l}\text { Postura ante el } \\
\text { objeto }\end{array}$ & $\begin{array}{c}\text { Convicción } \\
\text { metodológica }\end{array}$ & $\begin{array}{c}\text { Compromiso } \\
\text { ontológico }\end{array}$ & $\begin{array}{c}\text { Dualidad de la } \\
\text { estructura }\end{array}$ & Dualismo analítico \\
\hline Tradición filosófica & Positivismo & Empirismo & Estructuración & Emergentismo \\
\hline Unidad & $\begin{array}{c}\text { Propiedades } \\
\text { colectivas }\end{array}$ & Elementos últimos & Estructuras & Procesos \\
\hline Foco temporal & $\begin{array}{c}\text { T1-T3 } \\
\text { Structural } \\
\text { conditioning }\end{array}$ & $\begin{array}{c}\text { T2-T4 } \\
\text { Structural } \\
\text { elaboration }\end{array}$ & $\begin{array}{c}\text { T2-T3 } \\
\text { Social interaction }\end{array}$ & $\begin{array}{c}\text { T1-T4 } \\
\text { Three parts cycle }\end{array}$ \\
\hline
\end{tabular}

La tabla 1 permite reconstruir el movimiento que va de la crítica general al conflacionismo a la proposición de un realismo crítico morfogenético. Tal reconstrucción deja entrever varios aspectos interesantes, primero, muestra una estrategia Mp compleja, marca por la distinción conflación/no-conflación, que articula crítica general del conflacionismo y una alternativa programática a ella asentada en la categoría de morfogénesis no-conflacionista; segundo, construye para el programa un conjunto de antecedentes disciplinarios (Lockwood, Buckley) e interdisciplinarios (filosóficos: Bhaskar, Harré, Bunge; cibernéticos: Maruyama), que impiden su aislamiento y la reconectan con otras investigaciones, nutriendo el fundamento operativo. El tránsito hacia la morfogénesis social; tercero, encuentra en el concepto de mecanismo generativo, que re-especificará sociológicamente como "práctica social", la opción para aunar la designación de la unidad de análisis y de la infraestructura dualista de niveles; cuarto, propone alternativas para cada uno de los ejes diagnosticados, sin romper con ellos, el resultado es una posición novedosa e innovadora punto por punto, pero prescinde de la semántica del "cambio de paradigma", tan en boga en la sociología continental y trasatlántica de los años setenta y ochenta, que buscaba por esa vía la salida a la crisis de "las dos sociologías". 
Consideramos, a modo de cierre, de relevancia heurística iniciar las tareas de comparación programática entre este diagnóstico y otros esfuerzos coetáneos de igual naturaleza, y encontramos fundamental, planteadas ya las equivalencias funcionales, iniciar las tareas de comparación y análisis sistemáticos de este fundamento operativo con los de otros programas teóricos igualmente innovadores y fundamentales en la posguerra tardía.

\section{Bibliografía}

Archer, M. 1985. The myth of cultural integration. The British Journal of Sociology 36(3): 333-353. https://doi.org/10.2307/590456

Archer, M. 1995. Realist social theory. The morphogenetic approach. Cambridge: Cambridge University Press

Archer, M. 1996. Culture and agency. The place of culture in social theory. Cambridge: Cambridge University Press.

Archer, M. 2000a. Being human: the problem of agency. Cambridge: Cambridge University Press.

Mascareño, A. 2008. Acción, estructura y emergencia en la teoría sociológica. Revista de Sociología 22:

217-256. https://revistadesociologia.uchile.cl/index.php/RDS/article/view/14492

Recibido el 28 Mar 2018

Aceptado el 16 Jun 2018 